\title{
Branching ratios for the decay of $d^{*}(2380)$
}

\author{
M. Bashkanov ${ }^{1,2,3}$, H. Clement ${ }^{2,3, a}$, and T. Skorodko ${ }^{4}$ \\ 1 School of Physics and Astronomy, University of Edinburgh, James Clerk Maxwell Building, Peter Guthrie Tait Road, \\ Edinburgh, EH9 3FD, UK \\ 2 Physikalisches Institut, Eberhard-Karls-Universität Tübingen, Auf der Morgenstelle 14, D-72076 Tübingen, Germany \\ 3 Kepler Center for Astro and Particle Physics, University of Tübingen, Germany \\ 4 Department of Physics, Tomsk State University, 36 Lenina Avenue, Tomsk, 634050, Russia
}

Received: 15 April 2015 / Revised: 24 June 2015

Published online: 20 July 2015

(C) The Author(s) 2015. This article is published with open access at Springerlink.com

Communicated by P. Salabura

\begin{abstract}
Based on measurements, the branching ratios for the decay of the recently discovered dibaryon resonance $d^{*}(2380)$ into two-pion production channels and into the $n p$ channel are evaluated. Possibilities for a decay into the isoscalar single-pion channel are discussed. Finally, the electromagnetic decay of $d^{*}(2380)$ is considered.
\end{abstract}

\section{Introduction}

Recent WASA experiments at CELSIUS [1] and COSY [2, $3]$ on the basic double-pionic fusion to deuterium identified a narrow isoscalar resonance structure with mass $m \approx 2.37 \mathrm{GeV}$ and width $\Gamma \approx 70 \mathrm{MeV}$ in the total cross section of the reactions $p n \rightarrow d \pi^{0} \pi^{0}$ and $p n \rightarrow d \pi^{+} \pi^{-}$. The differential distributions are consistent with a spinparity assignment of $J^{P}=3^{+}$to this structure. Subsequent measurements of two-pion production reactions, where the two colliding nucleons do not fuse to deuterium, but stay unbound, also show this resonance effect, if the reaction contains isoscalar parts [4-6].

The final proof for this structure to represent a genuine $s$-channel resonance has been achieved by polarized $\vec{n} p$ scattering in the energy region of interest. The obtained analyzing power data produce a pole in the coupled ${ }^{3} D_{3}{ }^{3} G_{3}$ partial waves at $(2380 \pm 10)-i(40 \pm 5) \mathrm{MeV}$, if included in the SAID data base with subsequent partialwave analysis [7-9]. Henceforth, this state has been denoted by $d^{*}(2380)$ following the convention used for nucleon excitations.

The golden reaction channel for the observation of $d^{*}(2380)$ turned out to be $p n \rightarrow d \pi^{0} \pi^{0}$, since there the background from conventional processes due to $t$-channel Roper and $\Delta \Delta$ excitations is smallest. Since WASA has been the only detector with a nearly full solid angle coverage for both charged and neutral particles, which was placed at a hadron accelerator, it is of no surprise that there were no data for this channel from previous measurements. Thus it was left to the WASA Collaboration

\footnotetext{
a e-mail: clement@pit.physik.uni-tuebingen.de
}

to reveal the pronounced Lorentzian energy dependence sitting upon an only small background in the total cross section of this channel.

The experimental Dalitz plot at resonance in this golden channel $p n \rightarrow d \pi^{0} \pi^{0}$ points to a $\Delta \Delta$ excitation in the intermediate state [2], which means that $d^{*}(2380)$ behaves asymptotically like a bound $\Delta \Delta$ system. We note that already in 1964 Dyson and Xuong [10] predicted exactly such a state, based on $S U(6)$ symmetry considereations, with a value for the mass remarkably close to the one of the now observed dibaryon resonance. Later on Kamae and Fujita [11] as well as Goldman et al. [12] predicted such a state, though the latter with a markedly lower mass. Only recent modern quark-model calculations see this state properly near the experimental mass [13-17]. Also new relativistic Faddeev-type calculations carried out by Gal and Garcilazo by use of hadronic interactions find this state at the correct mass $[18,19]$.

If we account just for the well-known momentum dependence of the width of the $\Delta(1232)$ resonance, then we expect for a conventional $\Delta \Delta$ system bound by $80 \mathrm{MeV}$ a decay width of about $160 \mathrm{MeV}[20,21]$. This is more than twice that observed for $d^{*}(2380)$. Hence it is of no surprise that theoretical calculations predict a too large width for this state. Until very recently Gal and Garcilazo [19] came closest with about $100 \mathrm{MeV}$ for the width, if they allow for all decay channels discussed in the next section. This discrepancy in the width might indicate some exotic contribution, which hinders the decay of $d^{*}(2380)$ — such as hidden color as discussed in refs. $[15,20]$. In fact, very recently it has been shown in ref. $[21,22]$ that the experimentally observed small width can be reconciled theoretically, if the hidden color aspect is taken into account. 
To clarify the experimental situation, we examine in the following, whether all major hadronic decays of $d^{*}(2380)$ have been identified and understood or whether a substantial decay branch has escaped detection so far.

\section{Decay channels and widths}

\section{Hadronic decays}

We consider the following reaction scenario as suggested by the data on two-pion production $[2-6]$ :

$$
p n \rightarrow d^{*}(2380) \rightarrow \Delta \Delta \rightarrow(N N \pi \pi)_{I=0},
$$

where $d^{*}(2380)$ denotes an $s$-channel resonance both in $p n$ and $\Delta \Delta$ systems. By this scenario, we neglect a possible direct decay $d^{*}(2380) \rightarrow N N \pi$, but we shall come back to this point in the next to next section. Note that an intermediate $N \Delta$ configuration is excluded by isospin.

\section{$N N \pi \pi$ and $n p$ channels}

First, we consider the possible decay channels in the scenario of eq. (1). In particular we estimate the partial decay width into the elastic $p n$ channel.

The cross section of the isoscalar two-body resonance process $p n \rightarrow d^{*}(2380) \rightarrow \Delta \Delta$ is given by the relativistic Breit-Wigner formula [23]:

$$
\sigma_{p n \rightarrow \Delta \Delta}=\frac{4 \pi}{k_{i}^{2}} \frac{2 J+1}{\left(2 s_{p}+1\right)\left(2 s_{n}+1\right)} \frac{m_{d^{*}}^{2} \Gamma_{i} \Gamma_{f}}{\left(s-m_{d^{*}}^{2}\right)^{2}+m_{d^{*}}^{2} \Gamma^{2}},
$$

where $k_{i}$ denotes the initial center-of-mass momentum.

As best estimates for mass and width of the resonance, we take the average over the results from elastic scattering and two-pion production, i.e. $m_{d^{*}}=2.375 \mathrm{GeV}$ and $\Gamma=$ $75 \mathrm{MeV}$.

With $J=3$ and $s_{p}=s_{n}=1 / 2$, the peak cross section at $\sqrt{s}=m_{d^{*}}=2.375 \mathrm{GeV}\left(k_{i}=0.73 \mathrm{GeV} / c\right)$ is then

$$
\sigma_{p n \rightarrow \Delta \Delta}(\text { peak })=\sigma_{0} \frac{\Gamma_{i} \Gamma_{f}}{\Gamma^{2}},
$$

with

$$
\sigma_{0}=16.1 \mathrm{mb} \quad \text { (unitarity limit). }
$$

Since we also have

$$
\Gamma=\Gamma_{i}+\Gamma_{f},
$$

we get from (3) and (5):

$$
\Gamma_{i}=\Gamma\left(\frac{1}{2} \pm \sqrt{\frac{1}{4}-\frac{\sigma_{p n \rightarrow \Delta \Delta}(\text { peak })}{\sigma_{0}}}\right) .
$$

In general, partial and total widths are momentum dependent quantities. Therefore, branching ratios, as we will derive below for $\mathrm{d}^{*}(2380)$, have been defined by convention to be quoted at the Breit-Wigner resonance mass or at the resonance pole, see Particle Data Group recommendations [23]. In case of $\mathrm{d}^{*}(2380)$ both coincide within uncertainties. Hence, we need to consider the resonance cross sections only at its peak position. Also, in all relevant WASA experiments the energy resolution has been similar, in the range of $10-20 \mathrm{MeV}$, which is small compared to the resonance width. Hence the smearing effect on the resonance curve is small and in particular comparable in all measured channels, so that this effect tends to cancel in the branching ratios to large extent.

To estimate $\sigma_{p n \rightarrow \Delta \Delta}($ peak $)$ consider the total cross sections of all channels, where the isoscalar $\Delta \Delta$ system can decay into:

i) $d \pi^{0} \pi^{0}$ and $d \pi^{+} \pi^{-}$:

Due to isospin rules we expect

$$
\sigma_{d \pi^{+} \pi^{-}}\left(d^{*}\right)=2 \sigma_{d \pi^{0} \pi^{0}}\left(d^{*}\right) .
$$

However, due to the isospin violation in the pion mass, the available phase space is somewhat smaller for charged pion production than for the production of the lighter neutral pions. In ref. [3] it has been shown that this results in a resonance cross section, which is lower by about $20 \%$ in case of the $d \pi^{+} \pi^{-}$channel. Hence we have

$$
\begin{aligned}
\sigma_{a} & :=\sigma_{d \pi^{+} \pi^{-}}\left(d^{*}\right)+\sigma_{d \pi^{0} \pi^{0}}\left(d^{*}\right) \\
& \approx 2.6 \sigma_{d \pi^{0} \pi^{0}}\left(d^{*}\right) .
\end{aligned}
$$

The peak cross section of the $p n \rightarrow d \pi^{0} \pi^{0}$ reaction at $\sqrt{s}=2.37 \mathrm{GeV}$ has been measured to be $0.27 \mathrm{mb}[3]$. This includes the contributions of the $t$-channel $\Delta \Delta$ and Roper excitations. Accounting for these background effects, the pure resonance cross section in this channel amounts to about $0.24 \mathrm{mb}$, i.e., $\sigma_{a} \approx 0.62 \mathrm{mb}$. Since the cross sections of the three fusion reactions $p n \rightarrow d \pi^{0} \pi^{0}, p n \rightarrow d \pi^{+} \pi^{-}$and $p p \rightarrow d \pi^{+} \pi^{0}$ measured in ref. [3] are closely connected by isospin, only a small uncertainty of about $10 \%$ in absolute normalization has been quoted. This leads to

$$
\sigma_{a} \approx 0.62(6) \mathrm{mb}
$$

ii) $p p \pi^{0} \pi^{-}, n n \pi^{+} \pi^{0}, n p \pi^{0} \pi^{0}$ and $n p \pi^{+} \pi^{-}$-isoscalar parts:

First, we consider the $p p \pi^{0} \pi^{-}$channel. Though both the $p p$ pair and the $\pi^{0} \pi^{-}$pair are isovector pairs, together they may couple to total isospin $I=0$. Hence the isoscalar resonance $d^{*}(2380)$ may also decay into the isoscalar part of the $p p \pi^{0} \pi^{-}$channel. In fact, the decay of the resonance into the $p p \pi^{0} \pi^{-}$channel proceeds via the same intermediate $\Delta^{+} \Delta^{0}$ system as the $d \pi^{0} \pi^{0}$ channel does. From isospin coupling we expect that the resonance decay into the $p p \pi^{0} \pi^{-}$system should be half of that into the $n p \pi^{0} \pi^{0}$ system, which is in the order of $0.2 \mathrm{mb}$ - see next to next paragraph. In fact, a recent measurement [4] of this channel 
by WASA-at-COSY is in agreement with a resonance contribution of $0.10(1) \mathrm{mb}$ in the total cross section at $\sqrt{s}=2.37 \mathrm{GeV}$.

The $n n \pi^{+} \pi^{0}$ channel is just the isospin mirrored one to the $p p \pi^{0} \pi^{-}$channel. Hence it has to have the same resonance contribution.

In a recent paper [24], Fäldt and Wilkin present an estimate of the resonance cross section in the $p n \rightarrow p n \pi^{0} \pi^{0}$ reaction. According to their calculation based on final state interaction theory, the expected peak cross section in the deuteron breakup channel $p n \pi^{0} \pi^{0}$ is about $85 \%$ that of the non-breakup channel $d \pi^{0} \pi^{0}$, i.e. about $0.2 \mathrm{mb}$. Recently also Albaladejo and Oset [25] estimated the expected resonance cross sections in $p n \rightarrow p n \pi^{0} \pi^{0}$ and $p n \rightarrow p n \pi^{+} \pi^{-}$using a more elaborate theoretical procedure. Their result for the $p n \rightarrow p n \pi^{0} \pi^{0}$ channel is compatible with that from ref. [24]. In fact, a recent measurement at WASA [5] shows that the data are in accordance with a contribution of $d^{*}(2380)$ with a strength of $0.20(3) \mathrm{mb}$.

The resonance effect in the isoscalar part of the $n p \pi^{+} \pi^{-}$channel is composed of the configurations, where either both $n p$ and $\pi^{+} \pi^{-}$pairs couple each to $I=0$ or both pairs each to $I=1$. The first case gives just twice the contribution in the $n p \pi^{0} \pi^{0}$ channel. The latter case provides the same situation as in the $p p \pi^{0} \pi^{-}$channel. Hence, we have

$$
\begin{aligned}
\sigma_{n p \pi^{+} \pi^{-}}\left(d^{*}\right) & \approx 2 \sigma_{n p \pi^{0} \pi^{0}}\left(d^{*}\right)+\sigma_{p p \pi^{0} \pi^{-}}\left(d^{*}\right) \\
& \approx 0.50(8) \mathrm{mb} .
\end{aligned}
$$

Note that in these non-fusion channels there is no ABC effect, i.e. no low-mass enhancement in the $\pi \pi$ invariant mass spectra [4-6]. Hence the phase-space reduction due to different masses of charged and neutral pions as discussed above for the $d \pi^{+} \pi^{-}$channel does not play a significant role here.

Our estimate for the resonant $p n \rightarrow p n \pi^{+} \pi^{-}$cross section is in good agreement with that of ref. [25], where, however, only the contributions with $I=0$ coupled nucleon and pion pairs were considered. Our result also is compatible with available measurements for this channel, see ref. [6], including the newest results from HADES [26].

In total we have, from these four reactions,

$$
\begin{aligned}
\sigma_{b}:= & \sigma_{n p \pi^{+} \pi^{-}}\left(d^{*}\right)+\sigma_{n p \pi^{0} \pi^{0}}\left(d^{*}\right) \\
& +\sigma_{p p \pi^{0} \pi^{-}}\left(d^{*}\right)+\sigma_{n n \pi^{+} \pi^{0}}\left(d^{*}\right) \\
= & 0.50(8) \mathrm{mb}+0.20(3) \mathrm{mb} \\
& +0.10(1) \mathrm{mb}+0.10(1) \mathrm{mb} \\
= & 0.90(13) \mathrm{mb} .
\end{aligned}
$$

Altogether we get as an estimate

$$
\sigma_{p n \rightarrow \Delta \Delta}(\text { peak })=\sigma_{a}+\sigma_{b} \approx 1.5(2) \mathrm{mb} .
$$

Putting this into eq. (6) and selecting the minus sign in front of the root (see discussion below) we obtain

$$
\Gamma_{i}=8(1) \mathrm{MeV}, \text { for } \Gamma=75 \mathrm{MeV},
$$

Table 1. Experimental branching ratios (BR) of the $d^{*}$ resonance into its decay channels based on eqs. (1)-(3) and (12) and the peak cross sections given under i) and ii).

\begin{tabular}{lll}
\hline Decay channnel & BR & Derived from \\
\hline$n p$ & $12(3) \%$ & measurement $[8]$ \\
$d \pi^{0} \pi^{0}$ & $14(1) \%$ & measurement $[3]$ \\
$d \pi^{+} \pi^{-}$ & $23(2) \%$ & measurement [3] \\
$n p \pi^{+} \pi^{-}$ & $30(5) \%$ & measurement $[6,26]$ \\
$n p \pi^{0} \pi^{0}$ & $12(2) \%$ & measurement [5] \\
$p p \pi^{0} \pi^{-}$ & $6(1) \%$ & measurement [4] \\
$n n \pi^{+} \pi^{0}$ & $6(1) \%$ & isospin symmetry \\
\hline
\end{tabular}

which in turn corresponds to a resonance cross section in the elastic $p n$ channel of only

$$
\sigma_{p n \rightarrow p n} \approx 0.17(2) \mathrm{mb},
$$

if we apply eq. (3) for the incident channel.

From the SAID partial-wave analysis of elastic $n p$ scattering including the new WASA data on polarized $\vec{n} p$ scattering in the energy region of $d^{*}(2380)$ an elastic partial width of $\Gamma_{i}=10(2) \mathrm{MeV}$ has been derived corresponding to a branching ratio of $12(3) \%$ [8]. This result agrees reasonably well with the value obtained above in eq. (13).

We note in passing that the other solution of eq. (6) - the one with the plus sign - leads to the complementary result, namely $\Gamma_{i}=67 \mathrm{MeV}$ - thus implying that the resonance would be predominantly elastic, i.e., mainly decay into the elastic channel and only weakly into the pionproduction channels. This solution is at obvious variance with elastic $n p$ scattering data.

From the peak cross sections given under i) and ii) as well as from eqs. (3)-(12) we may readily calculate the branching ratios $\mathrm{BR}:=\Gamma_{j} / \Gamma$ for the decay of the resonance into the individual $N N \pi \pi$ channels. The results are listed in table 1.

The experimental branching ratios listed in table 1 add up to $103(15) \%$, which is consistent within the assumption in eq. (1) that the $d^{*}$ decay proceeds solely via the intermediate $\Delta \Delta$ system and the $n p$ channel. From the data on differential observables, in particular Dalitz plots and $N \pi$-invariant mass spectra, we know that these are the dominant decay channels. But this does not exclude that there might be other small decay channels. E.g., it has been proposed that there might be a $5 \%$ decay branch $d^{*} \rightarrow d \sigma$ with an unusual light and narrow $\sigma$ particle, in order to describe the low-mass enhancement (ABC effect), see refs. [27,28].

From the fact that we have two independent results for the elastic partial width, namely the one obtained from the SAID partial-wave analysis and the one derived in eq. (8), we may obtain an upper limit for decay branchings not covered by eq. (1). If we account for such branching by a partial width $\Gamma_{s}$, then $\Gamma$ in eq. (6) is to be replaced by $\left(\Gamma-\Gamma_{s}\right)$. Assuming then, e.g., $\Gamma_{s} / \Gamma$ to be in the order of $10 \%$ leads subsequently to a reduction of our result for the 

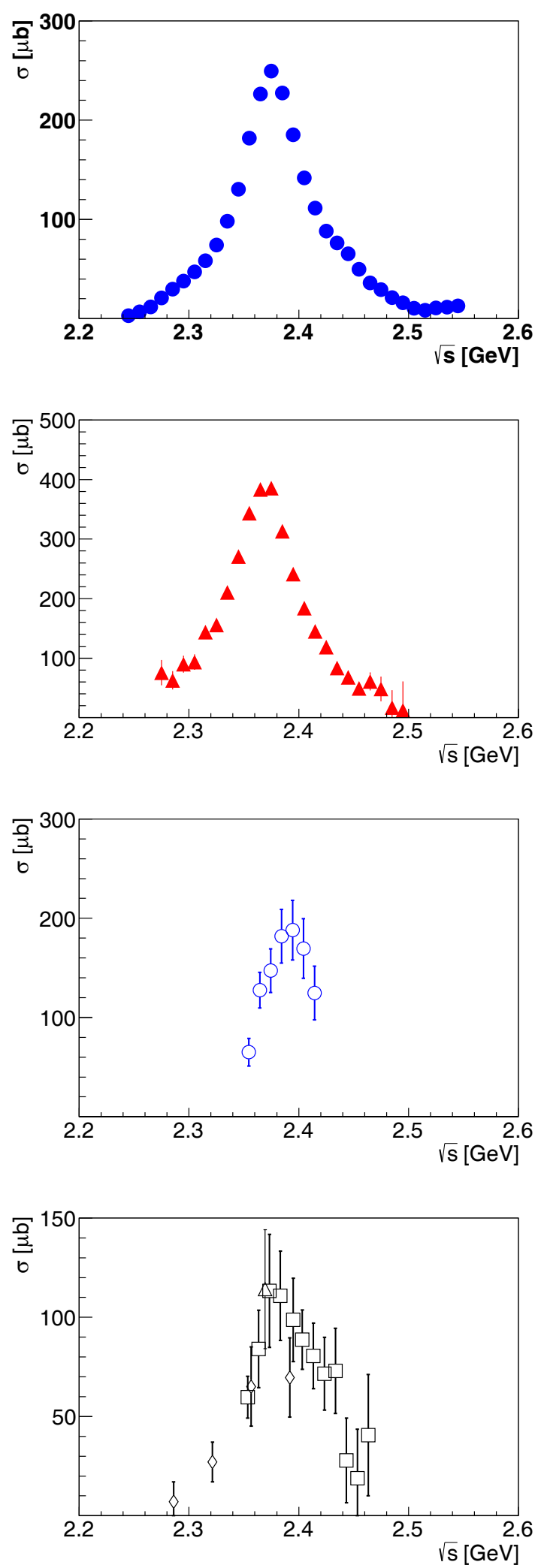

Fig. 1. Background-subtracted cross sections for the reactions $p n \rightarrow d \pi^{0} \pi^{0}, p n \rightarrow d \pi^{+} \pi^{-}$(isoscalar part), $p n \rightarrow p n \pi^{0} \pi^{0}$ and $p n \rightarrow p p \pi^{0} \pi^{-}$(from top to bottom) as obtained from WASA measurements $[2,3,5,4]$ and refs. $[29,30]$ by subtraction of the conventional background as given in refs. $[2,3,5,4]$. elastic partial width from 8 to $7 \mathrm{MeV}$, i.e., the agreement with the SAID results fades away. Thus we estimate that the sum of branchings missed by eq. (1) cannot be larger than $10 \%$.

As an alternative to the consideration of peak cross sections we may also exam the full resonance effect in the various reaction channels. Since the signal-to-background ratio is largest at the resonance peak, an integration over the full resonance region is naturally more sensitive to the assumed background and also to the negligence of interferences of the resonance with the background. However, statistics improves that way and this method is largely independent of the experimental energy resolution. Hence this method may serve as a cross-check of the results obtained by the peak cross section method. Figure 1 shows the background-subtracted cross sections for the reactions $p n \rightarrow d \pi^{0} \pi^{0}, p n \rightarrow d \pi^{+} \pi^{-}$(isoscalar part), $p n \rightarrow p n \pi^{0} \pi^{0}$ and $p n \rightarrow p p \pi^{0} \pi^{-}$(from top to bottom) as obtained from WASA measurements $[2,3,5,4]$ and refs. $[29,30]$ by subtraction of the conventional background given in refs. [2, $3,5,4]$. In order to keep the background problem as small as possible, but to account still sufficiently for any energy smearing due to the experimental resolution, we do not integrate over the full resonance region, but only from $m_{d^{*}}-\Gamma / 2$ to $m_{d^{*}}+\Gamma / 2$. If we normalize then to the branching ratio of $14 \%$ for the $d^{*}$ decay into $d \pi^{0} \pi^{0}$ we obtain $21(1) \%, 11(1) \%$ and $7(1) \%$ for the branching ratios the $d^{*}$ decay into $d \pi^{+} \pi^{-}$, pn $\pi^{0} \pi^{0}$ and $p p \pi^{0} \pi^{-}$channels, respectively. These results agree very well with those from the peak cross section method given in table 1 .

\section{$N N \pi$ channel}

One such channel, which has not yet been investigated experimentally, is $d^{*}(2380) \rightarrow N N \pi$, i.e. concerns isoscalar single-pion production. Since single-pion production in $N N$ collisions is either purely isovector or isospin mixed, the isoscalar part has to be obtained by combination of various cross section measurements. Most favorable appears the combination [31]:

$$
\sigma_{N N \rightarrow N N \pi}(I=0)=3\left(2 \sigma_{n p \rightarrow p p \pi^{-}}-\sigma_{p p \rightarrow p p \pi^{0}}\right),
$$

which derives from isospin decomposition of single-pion production [32]. Experimentally the most difficult part is the measurement of the $n p \rightarrow p p \pi^{-}$reaction, since it affords either neutron beam or target. Technically, this may be achieved by use of deuteron beam or target and measurement of the above reaction in the quasi-free mode. Since this necessitates, however, exclusive and kinematically complete measurements, in order to obtain reliable results, the data base on that is sparse, see ref. [31]. In particular, there are no data in the region of the $d^{*}(2380)$ resonance.

Also theoretically, it is difficult to construct a process of sizable cross section, where $d^{*}(2380)$ can decay into the single-pion channel. As mentioned at the beginning of this section, an intermediate $\Delta N$ system is isospin forbidden. Hence the next simple candidate configuration 
would be $N^{*}(1440) N$. However, spin-parity $J^{P}=3^{+}$of $d^{*}(2380)$ would require a $d$-wave between $N^{*}(1440)$ and $N$. Since the resonance energy is just at the $N^{*}(1440) N$ threshold, the probability for such a decay must be tiny already from the kinematical point of view [28]. Another possibility would be, if $d^{*}$ decays via the resonance structure $D_{12}(2150)$ with $I\left(J^{P}\right)=1\left(2^{+}\right)$at the $N \Delta$ threshold, as proposed in refs. $[18,19,27]$, which in turn can decay into the $N N$ system. This would give then the scenario $d^{*} \rightarrow D_{12}(2150) \pi \rightarrow N N \pi$. Also, the following process $n p \rightarrow d^{*} \rightarrow n p \rightarrow N N \pi$ appears to be possible in principle. However, aside from the fact this affords another reaction step beyond elastic scattering via the $d^{*}$ resonance, this process has to proceed with the ${ }^{3} D_{3}$ partial wave, which is not dominant in single pion production.

Whatsoever, a careful experimental investigation of this issue appears to be appropriate. Since WASA at COSY has finished its experimental program, dedicated measurements on that issue are no longer possible. However, the existing data base at WASA taken for various purposes contains data also on the desired single-pion production channels. A corresponding data analysis is in progress.

\section{Electromagnetic decays}

An electromagnetic excitation of the deuteron groundstate to the $d^{*}(2380)$ resonance is highly informative, since its transition formfactor gives access to size and structure of this resonance.

Judging just from the electromagnetic coupling constant, we expect electromagnetic decays to be suppressed already by two order of magnitudes - as is borne out, e.g. in the decay of the $\Delta$ resonance. A technical feasible excitation of $d^{*}(2380)$ would start by photo or electro excitation from the deuteron groundstate. A real or virtual photon would need then to transfer two units of angular momentum, i.e. be of $E 2$ or $C 2$ multipolarity, which lowers the transition probability further. In addition, the overlap in the wave functions of $d$ and $d^{*}(2380)$ enters profoundly. We are aware of two theoretical calculations dealing with such a scenario [33,34], where cross sections in the range $\mathrm{pb} / \mathrm{sr}-\mathrm{nb} / \mathrm{sr}$ are predicted for the forward angular range. These are two orders lower than conventional processes and hence it appears very difficult to sense the resonance excitation under usual conditions. In this respect, the reaction $\gamma d \rightarrow d \pi^{0} \pi^{0}$ appears to be attractive, since the conventional processes there are expected to be particularly small [35].

A way out could be polarization measurements. The situation looks similar to the one in elastic $n p$ scattering. As we have shown above, the $d^{*}(2380)$ resonance contribution is about $0.17 \mathrm{mb}$, which is more than two orders below the total elastic cross section. However, with help of the analyzing power, which consists only of interference terms in partial waves, it was possible to filter out reliably the resonance contribution.

The analogous case in electro or photo excitation of $d^{*}(2380)$ constitute measurements of the polarization of the outgoing proton in the reactions $\gamma d \rightarrow n \vec{p}$ and $\gamma^{*} d \rightarrow$ $n \vec{p}$, respectively, where $\gamma^{*}$ stands for a virtual photon created in inelastic electron scattering on the deuteron. As in the analyzing power of $n p$ scattering the angular dependence of the resonance effect in the polarization of the outgoing proton should be proportional to the associated Legendre polynomial $P_{3}^{1}(\cos \Theta)$ [8]. Therefore, the maximal resonance effect is expected to be at a scattering angle of $\Theta=90^{\circ}$.

In fact, such an effect has already been looked for previously by Kamae et al. in corresponding data from the Tokyo electron synchrotron [36-39]. In order to describe the observed large polarizations in the region of $d^{*}(2380)$ they fitted a number of resonances to the data, among others also a $J^{P}=3^{+}$state. However, presumably due to the limited data base they only obtained very large widths for these resonances in the order of $200-300 \mathrm{MeV}$ - as one would expect from conventional $\Delta \Delta$ excitations.

Recently, new polarization measurements from JLAB appeared [40]. Their lowest energy point is just in the $d^{*}(2380)$ region and is compatible with a maximal polarization of $P=-1$. It confirms thus the old Tokyo results in the sense that in this region there is a build-up of a very large polarization, which rapidly decreases both towards lower and higher energies, see fig. 1 in ref. [40]. Of course, a dedicated measurement over the region of interest is needed, in order to see, whether a narrow structure with the width of $d^{*}(2380)$ can be observed in this observable.

\section{Conclusions}

We have considered all $N N \pi \pi$ channels, into which the isoscalar dibaryon resonance $d^{*}(2380)$ can decay. For all of these channels there exist meanwhile experimental data, which show the $d^{*}(2380)$ resonance contribution and thus deliver the corresponding decay branchings. These branchings are compatible with what one expects from isospin coupling, if the intermediate state is a $\Delta \Delta$ configuration. This in turn agrees with the result from the Dalitz plot in the golden channel $d \pi^{0} \pi^{0}$, where the background situation is optimal. We add that there is no sign of this resonance observed in isovector $N N \pi \pi$ channels [3,41,42].

So the only possible hadronic decay channel, which missed so far a careful inspection, is the $N N \pi$ channel - though we know of no simple mechanism, by which $d^{*}(2380)$ could decay into such an isoscalar configuration. However, since such a scenario has not yet been examined experimentally, a dedicated experimental investigation appears to be in order.

The electromagnetic decay is expected to be tiny compared to the hadronic decay branchings. As we also pointed out, the $d^{*}(2380)$ contribution in deuteron disintegration processes will be even small compared to the background from conventional processes. A way out will possibly be the measurement of polarization observables. In particular, the polarization of the outgoing proton or neutron appears to be very promising as discussed above. Forthcoming measurements at MAMI could possibly give a decisive answer on that. 
We acknowledge valuable discussions on this matter with S.J. Brodsky, A. Gal, J. Haidenbauer, C. Hanhart, F. Hinterberger, T. Kamae, E. Oset, I. Strakovsky, G.J. Wagner, C. Wilkin, A. Wirzba, R. Workman and Z. Zhang. This work has been supported by the Forschungszentrum Jülich (COSY-FFE) and DFG (CL 214/3-1) as well as by STFC (ST/L00478X/1).

Open Access This is an open access article distributed under the terms of the Creative Commons Attribution License (http://creativecommons.org/licenses/by/4.0), which permits unrestricted use, distribution, and reproduction in any medium, provided the original work is properly cited.

\section{References}

1. M. Bashkanov et al., Phys. Rev. Lett. 102, 052301 (2009).

2. P. Adlarson et al., Phys. Rev. Lett. 106, 242302 (2011).

3. P. Adlarson et al., Phys. Lett. B 721, 229 (2013).

4. P. Adlarson et al., Phys. Rev. C 88, 055208 (2013).

5. P. Adlarson et al., Phys. Lett: B 743, 325 (2015).

6. H. Clement, M. Baskanov, T. Skorodko, Proc. STORI 2014, to be published in Phys. Scr., arXiv:1506.00557 [nucl-ex].

7. P. Adlarson et al., Phys. Rev. Lett. 112, 202301 (2014).

8. P. Adlarson et al., Phys. Rev. C 90, 035204 (2014).

9. R. Workman, EPJ Web of Conferences 81, 02023 (2014).

10. F.J. Dyson, N.-H. Xuong, Phys. Rev. Lett. 13, 815 (1964) 14, 339(E) (1965).

11. T. Kamae, T. Fujita, Phys. Rev. Lett. 38, 471 (1977).

12. T. Goldman et al., Phys. Rev. C 39, 1889 (1989).

13. H. Huang, J. Ping, F. Wang, Phys. Rev. C 89, 034001 (2014) and references therein.

14. Q.B. Li, P.N. Shen, J. Phys. G 26, 1207 (2000).

15. F. Huang, Z.Y. Zhang, P.N. Shen, W.L. Wang, arXiv: 1408.0458 [nucl-th].
16. X.Q. Yuan, Z.Y. Zhang, Y.W. Yu, P.N. Shen, Phys. Rev. C 60, 045203 (1999).

17. Hua-Xing Chen et al., Phys. Rev. C 91, 025204 (2015).

18. A. Gal, H. Garcilazo, Phys. Rev. Lett. 111, 172301 (2013).

19. A. Gal, H. Garcilazo, Nucl. Phys. A 928, 73 (2014).

20. M. Bashkanov, Stanley J. Brodsky, H. Clement, Phys. Lett. B 727, 438 (2013).

21. Yubing Dong, Pengnian Shen, Fei Huang, Zongye Zhang, Phys. Rev. C 91, 064002 (2015).

22. F.Huang, P.N. Shen, Y.-B. Dong, Z.Y. Zhang, arXiv: 1505.05395 [nucl-th].

23. Particle Data Group Collaboration (K.A. Olive et al.), Chin. Phys. C 38, 090001 (2014).

24. G. Fäldt, C. Wilkin, Phys. Lett. B 701, 619 (2011).

25. M. Albaladejo, E. Oset, Phys. Rev. C 88, 014006 (2013).

26. G. Agakishiev et al., arXiv:1503.04013 [nucl-ex].

27. M. Platonova, V. Kukulin, Phys. Rev. C 87, 025202 (2013).

28. M. Bashkanov, H. Clement, T. Skorodko, arXiv:1502. 07500 [nucl-ex].

29. T. Tsuboyama et al., Phys. Rev. C 62, 034001 (2000).

30. D.C. Brunt, M.J. Calyton, B.A. Westwood, Phys. Rev. 187, 1856 (1969).

31. V.V. Sarantsev et al., Eur. Phys. J. A 43, 11 (2010).

32. J. Bystricky et al., J. Phys. 48, 1901 (1987).

33. Chun Wa Wong, Phys. Rev. C 61, 064011 (2000).

34. Di Qing, He-Ming Sun, Fan Wang, Chin. Phys. Lett. 18, 885 (2001).

35. M. Egorov, A. Fix, Nucl. Phys. A 933, 104 (2015).

36. T. Kamae et al., Phys. Rev. Lett. 38, 468 (1977).

37. T. Kamae et al., Nucl. Phys. B 139, 394 (1978).

38. H. Ikeda et al., Phys. Rev. Lett. 42, 1321 (1979).

39. H. Ikeda et al., Nucl. Phys. B 172, 509 (1980).

40. K. Wijesooriya et al., Phys. Rev. Lett. 86, 2975 (2001).

41. T. Skorodko et al., Phys. Lett. B 679, 30 (2009).

42. T. Skorodko et al., Phys. Lett. B 695, 115 (2011). 